\title{
Correction to: Anatomical-positional relationship between the bone structure of the distal radius and flexor pollicis longus tendon using ultrasonography
}

\author{
Mayuko Kinoshita $^{1} \cdot$ Kiyohito Naito $^{1}$ D $\cdot$ Kenji Goto $^{1} \cdot$ Yoichi Sugiyama $^{1} \cdot$ Nana Nagura $^{1} \cdot$ Hiroyuki Obata $^{1} \cdot$ \\ Yoshiyuki Iwase $^{2} \cdot$ Kazuo Kaneko $^{1}$
}

Published online: 5 March 2022

(c) Springer-Verlag France SAS, part of Springer Nature 2022

\section{Correction to:}

\section{Surgical and Radiologic Anatomy (2019) 41:785-789} https://doi.org/10.1007/s00276-019-02216-9

In the original publication of the article, there are two errors.

We would like you to correct the following two amendments.

This is the first error in ethical approval ID that given in "Materials and methods" section.

This should be corrected as follows.

P 786, Line 2-4 in the original publication of the article

\section{Before correction}

The study was approved by the Ethics Committee for Medical Research of our university (No. 18-054), and informed consent was obtained from all volunteers.

\section{Correction}

The study was approved by the Ethics Committee for Medical Research of our university (No. 17-250), and informed consent was obtained from all patients.

The original article can be found online at https://doi.org/10.1007/ s00276-019-02216-9.

Kiyohito Naito

knaito@juntendo.ac.jp

1 Department of Orthopaedics, Juntendo University School of Medicine, 1-5-29 Yushima, Bunkyo-ku, Tokyo 113-0034, Japan

2 Department of Orthopaedic Surgery, Juntendo Tokyo Koto Geriatric Medical Center, 3-3-20 Shinsuna, Koto-ku, Tokyo 136-0075, Japan
This is the second error in the subject information that given in "Materials and methods" section.

This should be corrected as follows.

P 786, Line 5-11 in the original publication of the article

\section{Before correction}

The Japanese subjects were 16 healthy male volunteers (26 wrist joints) and 16 healthy female volunteers (30 wrist joints) with no past medical history of trauma or complaint of the wrist joint (56 wrist joints in total, 26 in male and 30 in female; mean age: $32.9 \pm 8.5$ ). Six wrists in male and two wrists in female were excluded in this study due to past traumatic history.

\section{Correction}

The Japanese subjects were 56 patients (26 in male and 30 in female; mean age: $32.9 \pm 8.5$ ) with distal radius fractures. The 56 non-injured side of wrists with no past medical history of trauma or complaint of the wrist joint were included in this study.

Publisher's Note Springer Nature remains neutral with regard to jurisdictional claims in published maps and institutional affiliations. 